\title{
Improved Finite Element Method for EMAT Analysis and Design
}

\author{
R. Jafari-Shapoorabadi, Student Member, IEEE, A. Konrad, Fellow, IEEE, and A. N. Sinclair, Member, IEEE
}

\begin{abstract}
Electromagnetic acoustic transducers (EMATs) operating in transmitting mode are examined. Two different finite element formulations, derived for two different definitions of source current density, are compared in order to show the importance of skin and proximity effects. An EMAT consisting of six source conductors is modeled as an example. Results obtained with an earlier method are compared with new FEM results at two different frequencies. The effect of lift-off and distance between conductors is investigated.
\end{abstract}

Index Terms-EMAT, finite element method, NDE, time domain analysis.

\section{INTRODUCTION}

$\mathbf{U}$ LTRASOUND as a means of searching for flaws in structural materials plays an important role in nondestructive testing/evaluation (NDT/NDE). A noncontact way of generating ultrasonic waves in an electrically conducting test specimen is by means of EMATs. EMATs are used not only for flaw characterization but also for thickness measurements and the study of many fundamental physical properties of linear and nonlinear materials. Their operation is based on the combination of eddy currents with ultrasound techniques. Although EMATs present several advantages for ultrasonic NDT, the efficiency of the transduction mechanism is low. Thus, design optimization of EMAT configurations is desirable to achieve an acceptable signal-to-noise ratio.

Transient electromagnetic field effects in EMATs consist of skin and proximity effects and eddy current phenomena, which are governed by the diffusion equation. In earlier works [1]-[3], the two-dimensional (2D) finite element method (FEM) was used to solve for the magnetic vector potential (MVP) for a given source current density (SCD). In [1] and [2], the SCD is treated as a known function of total current. However, according to electromagnetic theory, the SCD is also a function of the time derivative of the MVP. In this paper, the complete equation for the SCD is considered and a 2D time-domain FEM is used to solve the resulting system, which now includes, in addition to eddy currents, skin and proximity effects.

Manuscript received October 13, 2000.

This work was supported by the Canadian Natural Sciences and Engineering Research Council (NSERC), ANDEC Manufacturing of Toronto, and the Ontario Centre for Automotive Materials and Manufacturing.

R. Jafari-Shapoorabadi and A. Konrad are with the Edward S. Rogers Sr. Department of Electrical and Computer Engineering, University of Toronto, Toronto, ON, Canada M5S 3G4 (e-mail: jafari@power.ele.utoronto.ca; a.konrad@utoronto.ca).

A. N. Sinclair is with the Department of Mechanical and Industrial Engineering, University of Toronto, Toronto, ON, Canada M5S 3G8 (e-mail: sinclai@mie.utoronto.ca).

Publisher Item Identifier S 0018-9464(01)07195-3.
In [1], an analytical solution has been obtained for a differential equation which is based on an incomplete equation for the $\mathrm{SCD}$. A time domain solution has been derived from a frequency domain solution by substitution of $j \omega$ with the Laplace variable $s$. In general, the steady-state solution in the frequency domain is equivalent to the particular solution in the time domain. The authors of [1] did not use initial conditions and did not compute the homogeneous solution of the differential equation.

In [6], the authors made use of 1D analytical solutions to validate FEM numerical results. In this paper, a practical EMAT example containing six source conductors is modeled. Results obtained with an earlier method are compared with new FEM results at two different frequencies. Finally, the effects of lift-off and distance between conductors on the Lorentz electromagnetic force inside a test sample are investigated.

\section{MAGNeTiC FIELD EQUATIONS}

The transient magnetic field for a transmitting EMAT may be stated in terms of the MVP and SCD by the following diffusion equation [4], [5]:

$$
-\operatorname{div} \frac{1}{\mu} \operatorname{grad} A_{z}+\sigma \frac{\partial A_{z}}{\partial t}=j_{s k}
$$

where $\mu, \sigma, A_{z}$ and $j_{s k}$ are the permeability, conductivity, MVP, and SCD of the $k$ th source conductor, respectively. In [4] and [5], the above equation is solved for a given SCD. In general, $A_{z}$ and $j_{s k}$ are unknown. On the other hand, in some applications the total currents are known. In [4] and [5], it is not clear how the SCD is determined from total current. For time domain field analysis just like in the case of static field analysis, the following simple equation for $j_{s k}$ is used [1], [2]:

$$
j_{s k}=\frac{i_{k}(t)}{S_{k}}
$$

where $i_{k}(t)$ and $S_{k}$ are the total current and cross-sectional area of the $k$ th source conductor, respectively. In this paper, the above equation is referred to as the incomplete equation for the SCD. By substituting (2) into (1), the differential equation becomes

$$
-\operatorname{div} \frac{1}{\mu} \operatorname{grad} A_{z}+\sigma \frac{\partial A_{z}}{\partial t}=\frac{i_{k}(t)}{S_{k}} .
$$

By using the integral form of Maxwell's second equation, one obtains a complete equation for $j_{s k}$ as follows:

$$
j_{s k}=\frac{i_{k}(t)}{S_{k}}+\frac{\sigma}{S_{k}} \frac{\partial}{\partial t} \iint_{R_{k}} A_{z} d s
$$


where $R_{k}$ represents the cross-sectional region of the $k$ th conductor. By substituting (4) into (1), the differential equation becomes

$$
\operatorname{div} \frac{1}{\mu} \operatorname{grad} A_{z}+\sigma \frac{\partial A_{z}}{\partial t}-\frac{\sigma}{S_{k}} \frac{\partial}{\partial t} \iint_{R_{k}} A_{z} d s=\frac{i_{k}(t)}{S_{k}} .
$$

\section{ACOUSTIC FIELD EQUATION}

The Lorentz force produces an acoustic wave inside the test material. This force is caused by the interaction between the electric current density, which is induced through the eddy current coil, and the static magnetic field. In general, the direction and intensity of the force $f_{L}$ is determined by the vector equation

$$
\vec{f}_{L}=\vec{J} \times \vec{B}_{0}
$$

where $\vec{J}$ and $\vec{B}_{0}$ represent total current density and static magnetic flux density, respectively. In general, $\vec{J}$ is given by

$$
\vec{J}=\vec{J}_{s}-\sigma \frac{\partial \vec{A}}{\partial t}
$$

It is assumed that $J$ has only a $z$-component, $J_{z}$, which can be computed from

$$
J_{z}=-\sigma \frac{\partial A_{z}}{\partial t}
$$

Note that $\vec{J}_{s}$ is zero inside the specimen. The static magnetic field inside the specimen is produced either by a permanent magnet or a DC electromagnet. The acoustic field equation can be stated in terms of a particle displacement vector $\vec{u}$ and the Lorentz force as follows:

$$
\mu \nabla \times \nabla \times \vec{u}-(\lambda+2 \mu) \nabla \nabla \cdot \vec{u}+\rho \frac{\partial^{2} \vec{u}}{\partial t^{2}}=\vec{f}_{L}
$$

where $\rho$ is mass volume density and $\lambda$ and $\mu$ are Lamé constants.

\section{Finite ELEMENT FoRMULATION}

The discretized finite element (FE) equivalent of (3) becomes [4]:

$$
\sigma T \frac{d A}{d t}+\frac{1}{\mu} S A=T J_{s}
$$

$S_{n \times n}$ and $T_{n \times n}$ are FE coefficient matrices. The discretized FE equivalent of (5) becomes [6]

$$
\sigma\left(T-Q P^{-1} Q^{T}\right) \frac{d A}{d t}+\frac{1}{\mu} S A=Q P^{-1} I(t) .
$$

To solve the ordinary differential equation (11), a pure implicit scheme is applied

$$
\left(\frac{R}{\Delta t}+\frac{1}{\mu} S\right) A_{n}=\frac{R}{\Delta t} A_{n-1}+Q P^{-1} I\left(t_{n}\right)
$$

where the matrix $R_{n \times n}$ is given by

$$
R=\sigma\left(T-Q P^{-1} Q^{T}\right) .
$$

It is simple to prove that $R$ is symmetric.

In (6), numerical results obtained with the above finite element formulation were shown to be in good agreement with 1D analytical results.

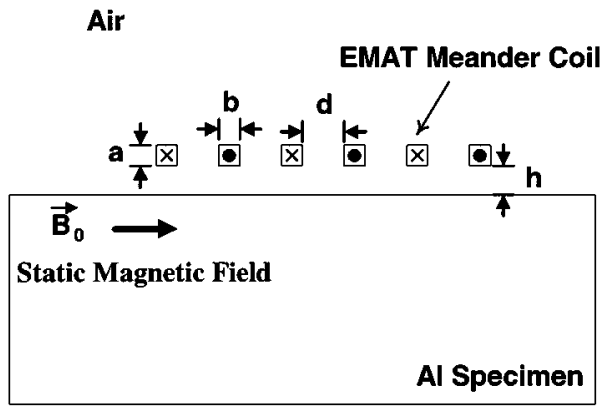

Fig. 1. Meander coil, static magnetic field and aluminum specimen.

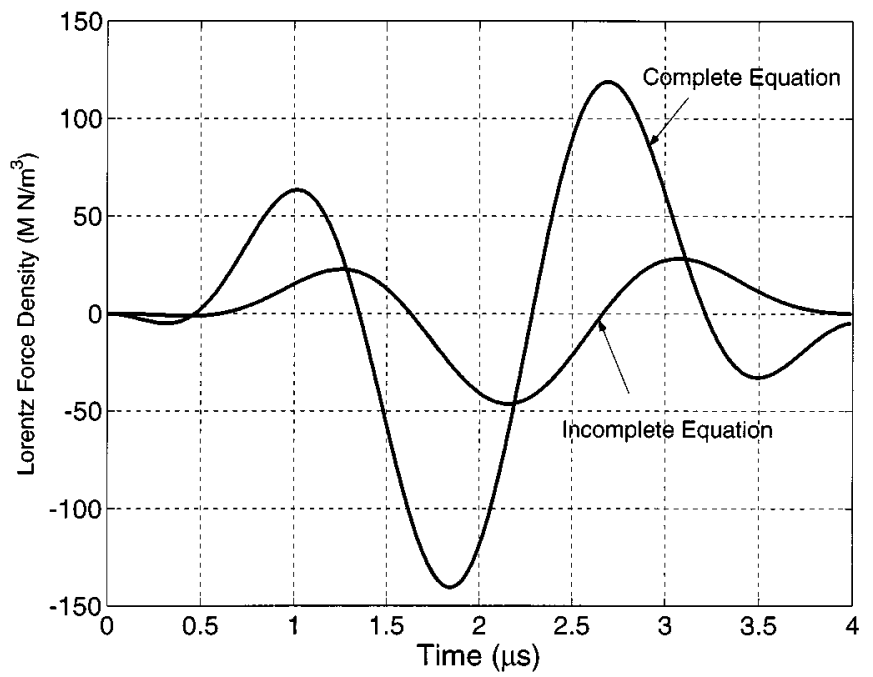

Fig. 2. Comparison of FEM results for force density at $500 \mathrm{kHz}$.

\section{EMAT EXAMPLE}

Fig. 1 depicts a meander coil EMAT configuration containing six identical conductors of rectangular cross section and dimension $a$ by $b$. Parameters $d$ and $h$ are the distance between the source conductors and lift-off, respectively. The transient excitation current of the $k$ th conductor, $i_{k}(t)$, is assumed to be given by

$$
i_{k}(t)= \begin{cases}(-1)^{k} I_{0}\left[1-\cos \left(\left(\omega_{0} t\right) / n\right)\right] \cos \left(\omega_{0} t\right) \\ \text { for } 0 \leq t \leq(2 n \pi) / \omega_{0} \\ 0 & \text { for } t \geq(2 n \pi) / \omega_{0}\end{cases}
$$

where$$
I_{0} \quad \text { is a constant current, }
$$$$
\omega_{0}=2 \pi f_{0} \quad \text { is the angular center frequency, and }
$$$$
n \quad \text { is the number of cycles. }
$$

The parameters used in this example are $f_{0}=500 \mathrm{KHz}, n=2$, $I_{0}=100 \mathrm{~A}, B_{0}=1 \mathrm{~T}, a=0.1 \mathrm{~mm}, b=0.5 \mathrm{~mm}, d=0.5 \mathrm{~mm}$ and $h=1 \mathrm{~mm}$. Meshes based on first-order triangular elements were used with the authors' FE codes to model the EMAT example. The meshes consist of about 800 nodes and 1500 elements. Identical meshes have been used for both formulations. The outer boundary is assumed to be a closed boundary, which is located sufficiently far from the sources.

Comparisons between FEM results obtained with the incomplete equation and the complete equation appear in Figs. 2 and 3 where the force density under the third source conductor from the right and near to the surface of an aluminum test sample is 


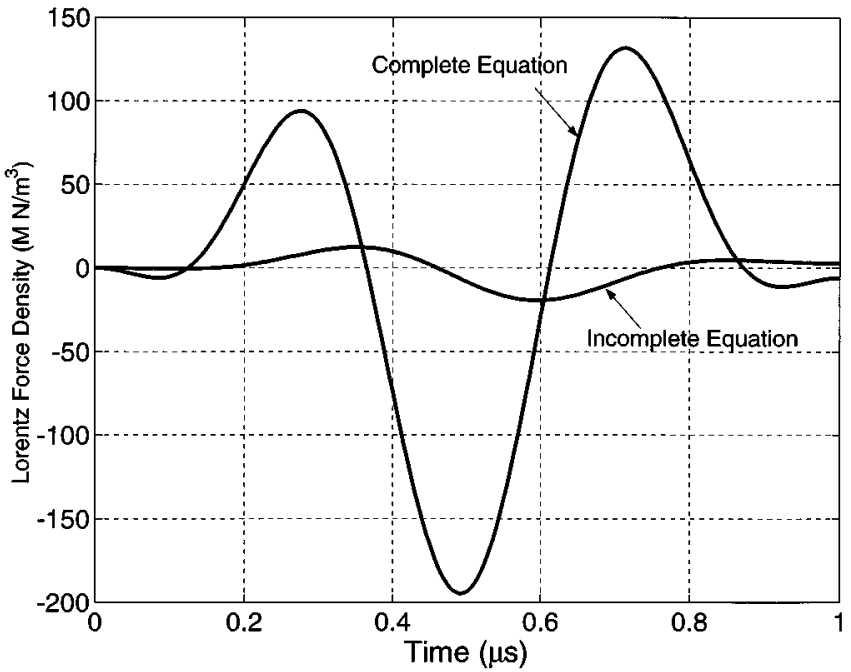

Fig. 3. Comparison of FEM results for force density at $2 \mathrm{MHz}$.

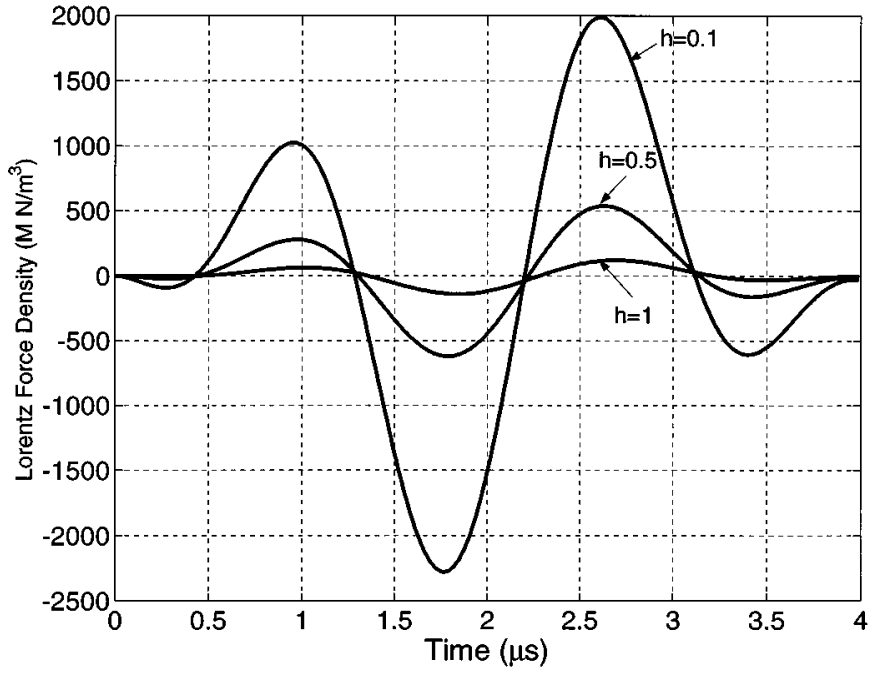

Fig. 4. FEM results based on the complete equation for the SCD: The effect of lift-off $h\left(f_{0}=500 \mathrm{kHz}, d=0.5 \mathrm{~mm}\right)$.

shown as a function of time. The effects of lift-off $h$ and the distance between conductors $d$ have been investigated. The results are shown in Figs. 4 and 5, respectively. They indicate that the force computed inside the specimen is a nonlinear function of the lift-off $h$ and the distance $d$. For example, Fig. 4 shows that decreasing the lift-off from $1 \mathrm{~mm}$ to $0.1 \mathrm{~mm}$ causes the force to become 16 times stronger. In practice, the lift-off depends on the temperature and surface condition of the specimen and can be as small as $100 \mu \mathrm{m}$. Also, Fig. 5 implies that increasing the distance $d$ increases the force. However, in practice, $d$ is determined by the wavelength of the desired acoustic wave.

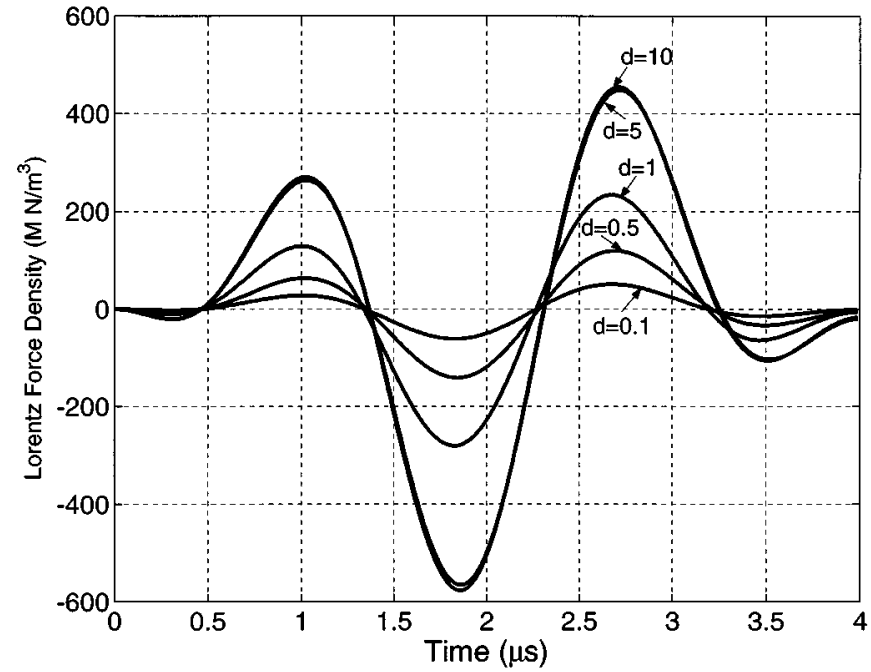

Fig. 5. FEM results based on the complete equation for the SCD: The effect of conductor spacing $d\left(f_{0}=500 \mathrm{kHz}, h=1 \mathrm{~mm}\right)$.

\section{CONCLUSIONS}

An EMAT example with six source conductors is modeled with 2D finite elements. FEM results are compared for both the incomplete and the complete equation for the SCD at two frequencies. Results show that when proximity and skin effects are ignored, errors as large as $75 \%$ in the magnitude of the generated ultrasonic wave may occur for $0.1 \mathrm{~mm} \times 0.5 \mathrm{~mm}$ rectangular conductors. The error increases with conductor dimensions and input current frequency. The effects of lift-off and distance between conductors are also shown for the EMAT example. The results indicate that the force computed inside the specimen is a nonlinear function of lift-off and the distance $d$ between the EMAT coil conductors.

\section{REFERENCES}

[1] R. Ludwig and X-W. Dai, "Numerical and analytical modeling of pulsed eddy currents in a conducting half-space," IEEE Trans. Magn., vol. MAG-26, no. 1, pp. 299-307, Jan. 1990.

[2] X-W. Dai, R. Ludwig, and R. Palanisamy, "Numerical simulation of pulsed eddy current nondestructive testing phenomena," IEEE Trans. Magn., vol. MAG-26, no. 6, pp. 3089-3096, Nov. 1990.

[3] M. Kaltenbacher, K. Ettinger, R. Lerch, and B. Tittmann, "Finite element analysis of coupled electromagnetic acoustic systems," IEEE Trans. Magn., vol. 35, no. 3, pp. 1610-1613, May 1999.

[4] P. P. Silvester and R. L. Ferrari, Finite Elements for Electrical Engineers. Cambridge, UK: Cambridge University Press, 1983.

[5] M. V. K. Chari and S. J. Salon, Numerical Methods in Electromagnetism. San Diego, CA: Academic Press, 2000.

[6] R. Jafari Shapoorabadi, A. N. Sinclair, and A. Konrad, "Finite element determination of the absolute magnitude of an ultrasonic pulse produced by an EMAT," in 2000 IEEE Int. Ultrasonics Symp., Puerto Rico, October 2000. 